\title{
A Comparison of Two Operation Methods Revealed the Risk Factors and the Necessity of LN-prRLN Dissection in Papillary Thyroid Carcinoma: A Retrospective Cohort Study in FUSCC
}

\author{
Yunjun Wang, ${ }^{1,2}$ Dezhi Wang, ${ }^{3}$ Lili Chen, ${ }^{1,2}$ Kai Guo $\mathbb{D}^{4},{ }^{4}$ and Tuanqi Sun $\mathbb{D}^{1,2}$ \\ ${ }^{1}$ Department of Head \& Neck Surgery, Fudan University Shanghai Cancer Center, Shanghai 200032, China \\ ${ }^{2}$ Department of Oncology, Shanghai Medical College, Fudan University, Shanghai 200032, China \\ ${ }^{3}$ Department of General Surgery, People's Hospital of Tongling, Anhui 244000, China \\ ${ }^{4}$ Department of Head \&Neck Surgery, Renji Hospital, School of Medicine, Shanghai Jiaotong University, Shanghai 200001, China
}

Correspondence should be addressed to Kai Guo; guokaics@hotmail.com and Tuanqi Sun; tuanqisun@126.com

Received 15 March 2020; Revised 20 July 2020; Accepted 21 August 2020; Published 10 September 2020

Academic Editor: Per Hellström

Copyright ( 2020 Yunjun Wang et al. This is an open access article distributed under the Creative Commons Attribution License, which permits unrestricted use, distribution, and reproduction in any medium, provided the original work is properly cited.

Background. Although the American Thyroid Association (ATA) guidelines indicate that central lymph nodes posterior to the right recurrent laryngeal nerve (LN-prRLN) should be routinely dissected, pr-RLN dissection is often neglected due to the high risk of injury to the recurrent laryngeal nerve (RLN). The purpose of this study was to investigate the risk factors associated with LN-prRLN metastasis in patients with papillary thyroid carcinoma (PTC) by preoperative examination and the indications for LN-prRLN dissection. Methods. A total of 1487 consecutive patients with PTC who underwent total thyroidectomy or right lobectomy plus isthmic resection with central LN dissection (CLND) were divided into two groups: patients with LN-prRLN dissection (group A) and patients without LN-prRLN dissection (group B). Clinicopathologic data were reviewed of the patients who were operated on by the same thyroid surgery team in the Department of Head Neck Surgery, Fudan University Shanghai Cancer Center (FUSCC) between August 2011 and May 2019. The relationships of LN-prRLN metastasis with clinicopathologic characteristics were analyzed by univariate and multivariate logistic regression. Results. The incidence of LN-prRLN metastasis was $34.1 \%(129 / 378)$. Univariate analysis showed that sex $(P \leq 0.001)$, tumor size $(P \leq 0.001)$, extrathyroidal extension $(P=0.002)$, concurrent Hashimoto's thyroiditis $(P=0.009)$, cLNMa (central lymph nodes anterior to the right recurrent laryngeal nerve) $(P \leq 0.001)$, cLNMa number $(P \leq 0.001)$, and lateral LN metastasis (LLNM) $(P \leq 0.001)$ were significantly associated with LNprRLN metastasis in PTC. Multivariate logistic regression analysis revealed that tumor size $(P=0.039)$, cLNMa $(P=0.001)$, and LLNM $(P=0.025)$ were independent risk factors for LN-prRLN metastasis in patients with PTC. Although there was no significant difference between the two groups in recurrence, we found that 4 cases relapsed in the LN-prRLN compartment in group B, while none relapsed in group A. Conclusion. LN-prRLN metastasis is often identified in patients with PTC. Patients with large tumor sizes, cLNMa and LLNM are at a high risk of LN-prRLN metastasis and should be recommended for careful LNprRLN dissection.

\section{Introduction}

Papillary thyroid cancer (PTC) is the most prevalent endocrine malignancy globally according to the cancer statistics data in China and SEER data (Surveillance, Epidemiology, and End Results 2004-2013) [1,2]. PTC often shows a better prognosis due to the slow development of characteristics, while lymph node metastasis has been reported to be as high as $20 \%-90 \%$, especially in the central compartment region [3-5]. Although the American Thyroid Association (ATA) and Chinese Thyroid Guidelines recommend routine (therapeutic and prophylactic) central lymph node dissection (CLND), approximately $10 \%$ of patients suffer from recurrence after the initial surgical treatment, and central neck recurrence accounts for $74 \%$ of all recurrent cases [6]. This suggests that there may be some problems in this process. 
According to ATA guidelines, the central compartment is subdivided into prelaryngeal (Delphian) lymph nodes, pretracheal lymph nodes, and paratracheal lymph nodes. The left recurrent laryngeal nerve (RLN) is located between the esophagus and trachea, but the right nerve ascends through the fat tissue of the right central compartment [7]. Due to anatomical differences, the RLN of the right side is more superficial than that of the left side. Some fat and lymphatic tissues are present posterior to the right RLN. Thus, the right paratracheal lymph node is further classified into two subregions by the right RLN [8]. The nodes located near the posterior side of the right nerve, upper to the esophagus and prevertebral fascia, are called the lymph nodes posterior to the right recurrent laryngeal nerve (LNprRLN), which were defined as VIb compartments, and lymph nodes anterior to the right recurrent laryngeal nerve were defined as VIa compartments [9]. The reported prevalence of LN-prRLN metastasis varies from 5.76\% to $26 \%[8,10-14]$. In particular, if minor lymph node metastasis (LNM) is located posterior to the right RLN, it is often neglected, and careful dissection is required because of the high likelihood of damage to the RLN. How to reconcile the protection of the RLN while thoroughly dissecting the central lymph nodes in certain situations is an urgent problem that requires exploration.

The purpose of this study was to investigate the frequency and risk factors related to LN-prRLN, evaluate recurrence, and assist surgeons in determining whether to perform selective LN-prRLN dissection in patients with PTC.

\section{Materials and Methods}

2.1. Patients. A total of 1487 patients with PTC who underwent thyroidectomy with LND in the Department of Head and Neck Surgery of Fudan University Shanghai Cancer Center (FUSCC) between August 2011 and May 2019 were retrospectively enrolled in this study. Preoperative assessment included ultrasonography (US), computed tomography (CT) scan, chest X-ray, and measurement of thyroglobulin (Tg), thyroid stimulating hormone (TSH), and anti-Tg antibody levels. US was preoperatively performed to assess the lymph node status and confirm no lymph node involvement in any of these patients. These patients underwent surgical treatment by one surgical team. Only newly diagnosed patients were enrolled. Patients with previous thyroid or parathyroid surgery, previous neck surgery, family history of cancer, and history of neck radiation were excluded. The study was approved by the Ethics Committees of Fudan University Shanghai Cancer Center, and all participants gave informed consent.

The clinicopathological characteristics of the different groups were compared, including sex, age, tumor size, location, multifocality, bilateral involvement, extrathyroidal extension, central lymph node metastasis (cLNM), central lymph nodes anterior to the right recurrent laryngeal nerve (cLNMa), and lateral lymph node metastasis (LLNM). Patients with at least one right lobe lesion were classified into the right lobe lesion group. For patients with multifocal tumors, the size and sublocation of the largest tumor were recorded for data analysis. The tumor size was extracted according to the longest diameter of the tumor and recorded in millimeters. In the patients who did not undergo lateral neck dissection, the number of metastatic lateral compartment lymph nodes was considered zero clinically.

2.2. Surgical Technique. All patients underwent total thyroidectomy or right lobectomy plus isthmus with routine CLND. LLNM ( $\mathrm{cN} 1 \mathrm{~b}$ ) was diagnosed based on preoperative image examination and fine-needle aspiration cytology or intraoperative frozen sectioning and sampling of suspicious lymph nodes, and patients with LLNM underwent lateral neck dissection.

The central compartment was defined according to the ATA's consensus of CLND [7]. The anatomic boundaries of the central compartment lymph node region were defined as follows: the carotid arteries formed the lateral boundaries, the tracheal margins formed the medial boundaries, the hyoid bone formed the superior boundary, the suprasternal fossa formed the inferior boundary, the anterior surface of thyroid gland formed the anterior boundary, and the prevertebral fascia formed the posterior boundary. The patients were divided into the following four groups according to Bae's method [8]: (1) Delphian lymph node; (2) right central lymph node; (3) left central lymph node; and (4) LN-prRLN. The pretracheal and paratracheal lymph nodes were combined into the central lymph node (CLN) group. LN-prRLN was analyzed separately. All lymph nodes were classified by the surgeon during the operation. The presence or absence of LNM was defined according to postoperative pathological reports.

2.3. Pathologic Examination. Postoperative histopathologic results were confirmed by 3 pathologists with over 10 years of experience at our institution. All cases were confirmed as PTC using intraoperative frozen paraffin sections and postoperative paraffin sections.

2.4. Follow-Up. Follow-up consisted of neck US examination, triiodothyronine (T3), tetraiodothyronine (T4), TSH, and thyroglobulin ( $\mathrm{Tg}$ ) every 3-6 months. If routine tests indicated recurrence, enhanced CT or FNA would be performed to confirm if additional surgery was needed. We considered the patients disease-free when serum Tg levels after injection recombinant human TSH (rhTSH) were $1 \mathrm{ng} /$ $\mathrm{ml}$, neck US was negative, and $\mathrm{Tg}$ antibody ( $\mathrm{TgAb}$ ) was undetectable.

2.5. Statistical Analysis. All statistical analyses were performed using Stata (version 12; Stata Corp, College Station, TX). Continuous variables were described using the mean \pm standard deviation (SD). Data were compared using Student's $t$-test. Categorical variables were described using frequency and percent and the chi-square test, and Fisher's exact test was used as the appropriate method to calculate the difference. Multiple logistic regression analysis was used 
to assess the statistical significance of the associations between LN-prRLN and clinicopathologic factors. Odds ratios (ORs) with 95\% relative confidence intervals were calculated to determine the relevance of all potential predictors. A $P$ value $<0.05$ was regarded as significant in the included studies.

\section{Results}

3.1. Patient Characteristics. Among the 1478 enrolled patients, the mean age at the first diagnosis was 46.14 years (range, 17-71 years). There were 353 men and 1134 women, with a female:male ratio of approximately $3.2: 1$. Overall, a right lobectomy was performed in 1016 (68.33\%) patients, and thyroidectomy was performed in 471 (31.67\%) patients. The detailed results are summarized in Table 1 . In particular, during the mean follow-up period of 36.9 months, recurrence was observed in $2.35 \%(35 / 1487)$ of patients.

Furthermore, these patients were divided into two groups: group A consisted of patients who underwent LNprRLN dissection, while group B did not. Table 2 demonstrates the clinicopathological characteristics of the two groups. Group A had a larger tumor size than group B $(P \leq 0.001)$. Concomitant Hashimoto's thyroiditis (co-HT) was found in $140(37.0 \%)$ and $227(20.47 \%)$ patients in these two groups, respectively $(P \leq 0.001)$. cLNMa, including positive and dissected cLNMa numbers (No.), were significantly higher in group A. Likewise, group A patients had, on average, more metastatic node numbers in the lateral neck $(P=0.022)$ than group $B$. The follow-up time was shorter in group $\mathrm{A}$. These results indicate that in recent years, we have tried to resect LN-prRLN conventionally in patients of a younger age, those with a larger tumor size, and those with more lymph node metastasis.

3.2. Predictors of LN-prRLN Metastasis. Next, we divided the 378 patients who underwent LN-prRLN dissection into two groups: LN-prRLN with positive LNM (LN-prRLNM+, $N=129$ ) and LN-prRLN with negative LNM (LNprRLNM-, $N=249)$. There were no significant differences in the factors of age $(P=0.481)$, multifocality $(P=0.255)$, concurrent nodular goiter $(P=0.152)$, and the size of cLNMa $(P=0.630)$ between the two groups. On univariate analysis, sex $(P \leq 0.001)$, tumor size $(P \leq 0.001)$, extrathyroidal extension (ETE) $(P=0.002)$, co-HT $(P=0.009)$, cLNMa and cLNMa numbers $(P \leq 0.001$ and $P \leq 0.001)$, and LLNM $(P \leq 0.001)$ were significantly correlated with LNprRLN metastasis. The mean numbers of metastatic CLNs in the LN-prRLNM+ and LN-prRLNM- groups were 3 (0-29) and $0(0-9)$, respectively $(P \leq 0.001)$. The mean tumor sizes were $13.9 \pm 9.1 \mathrm{~mm}$ and $9.6 \pm 6.3 \mathrm{~mm}$ in LN-prRLNM+ and LN-prRLNM-, respectively $(P \leq 0.001)$ (Table 3$)$. The results of the multivariable logistic regression analysis are shown in Table 4. Following adjustment for other predictors, sex $(\mathrm{OR}=0.61, P=0.116)$, extrathyroidal extension $(\mathrm{OR}=1.63$, $P=0.258)$, concurrent Hashimoto's thyroiditis $(\mathrm{OR}=0.62$, $P=0.104)$, and cLNMa number $(\mathrm{OR}=2.03, P=0.051)$ were no longer significantly predictive of LN-prRLN metastasis.
TABLE 1: Demographic characteristics of patients enrolled in this study.

\begin{tabular}{lc}
\hline Variables & $\begin{array}{c}\text { No. of patients }(\%) \\
(N=1487)\end{array}$ \\
\hline Age $(y$, mean \pm SD) & $46.14 \pm 11.42$ \\
Sex (Female/Male) & $1134(76.26 \%) / 353(23.74 \%)$ \\
Multifocality (yes/No) & $509(34.23 \%) / 978(65.77 \%)$ \\
Size (mm, mean \pm SD) & $9.70 \pm 6.73$ \\
Extensive invasion (yes/No) & $129(8.68 \%) / 1358(91.32 \%)$ \\
Co-HT (yes/No) & $367(24.68 \%) / 1120(75.32 \%)$ \\
Co-nodular goiter (yes/No) & $277(18.63 \%) / 1210(81.37 \%)$ \\
Thyroid operation methods & $1016(68.33 \%)$ \\
RL & $471(31.67 \%)$ \\
TT & \\
LNM & \\
cLNM (yes/No) & $074(45.33 \%) / 813(54.67 \%)$ \\
Positive cLNM no. (p50, (min- & $0(0-29)$ \\
max)) & $4(0-33)$ \\
Dissected cLNM no. (p50, (min- & \\
max)) & $140(9.41 \%) / 1347(90.59 \%)$ \\
LLNM (yes/No) & $4(0-37)$ \\
Positive LLNM no. (p50, (min- & \\
max)) & \\
Dissected LLNM no. (p50, (min- & $26.5(0-75)$ \\
max)) & $35(2.35 \%)$ \\
Recurrence (\%) & $36.91 \pm 21.43$ \\
Follow-up (months, mean \pm SD) &
\end{tabular}

Abbreviations: SD: standard deviation; Co-HT: concurrent Hashimoto's thyroiditis; Conodular goiter: concomitant with nodular goiter; LNM: lymph node metastasis; cLNM: central lymph node metastasis; LLNM: lateral lymph node metastasis.

However, tumor size $(\mathrm{OR}=1.15, \quad P=0.039)$, cLNMa $(\mathrm{OR}=1.28, P=0.001)$, and LLNM $(\mathrm{OR}=1.98, P=0.025)$ were significantly predictive of LN-prRLN metastasis.

3.3. Prognosis. To better understand the effect of LN-prRLN dissection on recurrence, we carefully compared the postoperative complications and recurrent locationsamong the reoperation patients. The postoperative hypocalcemia and hoarseness were more in group A, but most of them were temporary, and there was no difference in permanent. It may be related to the heavier and more thorough cleaning of LNprRLN (Table 5). The overall DFS was $97.65 \%$, and there was no significant difference between group $A$ and group $B$ $(P=0.460)$. The number of recurrent patients in the contralateral thyroid lobe, central compartment, and lateral compartment was $1,2,3$ and $3,10,16$, respectively. In particular, recurrence in the LN-prRLN region was 0 vs. 4 (Table 6). No significant difference was found (Figure 1), but there were still many relapses in LN-prRLN for patients who did not accept LN-prRLN dissection in the initial surgery. We have summarized the patients with relapse in the LNprRLN region, and the specific information is shown in Table 7. In general, the recurrence of this area is not rare. Figure 2 shows the CT and postoperative status of a patient with recurrence in the LN-prRLN region. The patient did not undergo LN-prRLN dissection in the first operation. LNprRLN was found on the right side, and a number of enlarged lymph nodes were found in the left lateral cervical 
TABLE 2: Demographic characteristics of patients enrolled in this study.

\begin{tabular}{|c|c|c|c|}
\hline \multirow{2}{*}{ Variables } & \multicolumn{2}{|c|}{ No. of patients (\%) } & \multirow{2}{*}{$P$ value } \\
\hline & Group A $(N=378)$ & Group B $(N=1109)$ & \\
\hline Age $(y$, mean $\pm S D)$ & $43.12 \pm 11.06$ & $47.17 \pm 11.36$ & $\leq 0.001$ \\
\hline Sex (Female/Male) & $291(77.0 \%) / 87(23.0 \%)$ & $843(76.0 \%) / 266(24.0 \%)$ & 0.702 \\
\hline Multifocality (yes/No) & $129(34.1 \%) / 249(65.9 \%)$ & $380(34.27 \%) / 729(65.73 \%)$ & 0.961 \\
\hline Size $(\mathrm{mm}$, mean $\pm \mathrm{SD})$ & $11.14 \pm 7.78$ & $9.23 \pm 6.33$ & $\leq 0.001$ \\
\hline Extensive invasion (yes/No) & $36(9.5 \%) / 342(90.5 \%)$ & $93(8.39 \%) / 1016(91.61 \%)$ & 0.497 \\
\hline Co-HT (yes/No) & $140(37.0 \%) / 238(63.0 \%)$ & $227(20.47 \%) / 882(79.53 \%)$ & $\leq 0.001$ \\
\hline Conodular goiter (yes/No) & $58(15.3 \%) / 320(84.7 \%)$ & $219(19.75 \%) / 890(80.25 \%)$ & 0.058 \\
\hline cN1a (Yes/No) & $96(25.4 \%) / 282(74.6 \%)$ & $332(30.0 \%) / 777(70.0 \%)$ & 0.100 \\
\hline \multicolumn{4}{|l|}{ LNM } \\
\hline cLNMa (yes/No) & $210(55.6 \%) / 168(44.4 \%)$ & $464(41.84 \%) / 645(58.16 \%)$ & $\leq 0.001$ \\
\hline cLNMa size $(\mathrm{mm}$, mean $\pm \mathrm{SD})$ & $7.86 \pm 5.31$ & - & \\
\hline Positive cLNMa no. (p50, (min-max)) & $1(0-29)$ & $0(0-17)$ & $\leq 0.001^{*}$ \\
\hline Dissected cLNMa no. (p50, (min-max)) & $5(0-33)$ & $4(0-25)$ & $\leq 0.001^{*}$ \\
\hline LLNM (yes/No) & $88(23.3 \%) / 290(76.7 \%)$ & $52(4.69 \%) / 1057(95.31 \%)$ & $\leq 0.001$ \\
\hline Positive LLNM no. (p50, (min-max)) & $5(1-37)$ & $3(1-33)$ & $0.022^{*}$ \\
\hline Dissected LLNM no. (p50, (min-max)) & $27(2-75)$ & $24.5(3-75)$ & $0.089^{*}$ \\
\hline LN-prRLN metastasis (yes/No) & $129(34.1 \%) / 249(65.9 \%)$ & - & - \\
\hline Size of LN-prRLN $(m m$, mean \pm SD $)$ & $5.82 \pm 3.60$ & - & - \\
\hline Recurrence $(\%)$ & $6(1.59 \%)$ & $29(2.61 \%)$ & 0.255 \\
\hline Follow-up (months, mean $\pm \mathrm{SD}$ ) & $23.19 \pm 16.09$ & $42.02 \pm 21.12$ & $\leq 0.001$ \\
\hline
\end{tabular}

SD: standard deviation; Co-HT: concurrent Hashimoto's thyroiditis; Conodular goiter: concomitant with nodular goiter; cN1a: clinical positive central lymph nodes metastasis; cLNMa:central lymph nodes anterior to the right recurrent laryngeal nerve; LNM: Lymph node metastasis; cLNM: Central lymph node metastasis; LLNM: Lateral lymph node metastasis. After the Shapiro-Wilk test and homogeneity of variance test, we found that the data did not conform to a normal distribution and that the variance was not uniform. Therefore, we used the Wilcoxon test to calculate the difference between the two groups.

TABLE 3: Univariate analysis of factors associated with LN-prRLN metastasis.

\begin{tabular}{|c|c|c|c|c|}
\hline Variables & $\begin{array}{c}\text { LN-prRLNM+ } \\
\quad(N=129)\end{array}$ & $\begin{array}{c}\text { LN-prRLNM- } \\
\quad(N=249)\end{array}$ & OR & $P$ value \\
\hline Age & $42.57 \pm 10.90$ & $43.41 \pm 11.15$ & $0.99(0.97-1.01)$ & 0.481 \\
\hline Sex (female $v s$. male) & $85(65.89 \%) / 44(34.11 \%)$ & $206(82.73 \%) / 43(17.27 \%)$ & $0.40(0.25-0.66)$ & $\leq 0.001$ \\
\hline Multifocality (yes vs. No) & $49(37.98 \%) / 80(62.02 \%)$ & $80(32.13 \%) / 169(67.87 \%)$ & $1.29(0.83-2.02)$ & 0.255 \\
\hline $\operatorname{Size}(\mathrm{mm})$ & $13.9 \pm 9.1$ & $9.6 \pm 6.3$ & $2.14(1.55-2.95)$ & $\leq 0.001$ \\
\hline Extrathyroidal extension (yes vs. No) & $21(16.28 \%) / 108(83.72 \%)$ & $15(6.02 \%) / 234(93.98 \%)$ & $3.03(1.51-6.11)$ & 0.002 \\
\hline Co-HT (yes vs. No) & $36(27.91 \%) / 93(72.09 \%)$ & $104(41.77 \%) / 145(58.23 \%)$ & $0.54(0.34-0.85)$ & 0.009 \\
\hline Co-nodular goiter (yes vs. No) & $15(11.63 \%) / 114(88.37 \%)$ & $43(17.27 \%) / 206(82.73 \%)$ & $0.63(0.34-1.18)$ & 0.152 \\
\hline \multicolumn{5}{|l|}{ Central LNM } \\
\hline cLNMa (yes vs. No) & $107(82.95 \%) / 22(17.05 \%)$ & $103(41.37 \%) / 146(58.63 \%)$ & $6.89(4.08-11.64)$ & $\leq 0.001$ \\
\hline cLNManumber & $3(0-29)$ & $0(0-9)$ & $1.56(1.39-1.75)$ & $\leq 0.001$ \\
\hline cLNMa size & $0.81 \pm 0.57$ & $0.77 \pm 0.51$ & $1.14(0.67-1.95)$ & 0.630 \\
\hline Lateral LNM (yes vs. No) & $53(41.09 \%) / 76(58.91 \%)$ & $35(14.06 \%) / 214(85.94 \%)$ & $4.26(2.58-7.04)$ & $\leq 0.001$ \\
\hline
\end{tabular}

Co-HT: concurrent Hashimoto's thyroiditis; Conodular goiter: concomitant with nodular goiter; cLNMa: central lymph nodes anterior to the right recurrent laryngeal nerve.

TABLE 4: Multivariate analysis of factors associated with LN-prRLN metastasis.

\begin{tabular}{lcc}
\hline Variables & OR & $P$ value \\
\hline Sex (Female $v s$. male) & $0.61(0.34-1.13)$ & 0.116 \\
Size & $1.45(1.02-2.06)$ & 0.039 \\
Extensive invasion (yes $v s$. No) & $1.63(0.70-3.84)$ & 0.258 \\
Co-HT (yes $v$ s. No) & $0.62(0.35-1.10)$ & 0.104 \\
cLNMa (yes $v$ s. No) & $1.28(1.11-1.48)$ & 0.001 \\
cLNMa number & $2.03(0.99-4.13)$ & 0.051 \\
Lateral LNM (yes $v s$. No) & $1.98(1.09-3.62)$ & 0.025 \\
\hline
\end{tabular}

Co-HT: concurrent Hashimoto's thyroiditis; cLNMa: central lymph nodes anterior to the right recurrent laryngeal nerve. 
TABLE 5: Comparisons of postoperative complications.

\begin{tabular}{lccc}
\hline Variables & $\begin{array}{c}\text { Group A } \\
(N=378)\end{array}$ & $\begin{array}{c}\text { Group B } \\
(N=1109)\end{array}$ & $P$ value \\
\hline $\begin{array}{l}\text { Postoperative hypocalcemia } \\
\text { Temporary }\end{array}$ & $28(7.4 \%)$ & $44(4.0 \%)$ & 0.012 \\
Persistent & $1(0.3 \%)$ & $3(0.3 \%)$ & 1.000 \\
\hline RLN monolateral palsy & & & \\
Temporary & $12(3.2 \%)$ & $26(2.3 \%)$ & 0.352 \\
Persistent & $3(0.8 \%)$ & $41(3.7 \%)$ & 0.586 \\
Chyle leakage & $18(4.8 \%)$ & $13(1.2 \%)$ & 0.362 \\
Hematoma & $2(0.5 \%)$ & $6(0.5 \%)$ & 0.380 \\
Wound infection & $3(0.8 \%)$ & & 0.701 \\
\hline
\end{tabular}

RLN: recurrent laryngeal nerve.

TABLE 6: Location of recurrence.

\begin{tabular}{lccc}
\hline Variables & $\begin{array}{c}\text { Group A } \\
(N=6)\end{array}$ & $\begin{array}{c}\text { Group B } \\
(N=29)\end{array}$ & $P$ value \\
\hline Contralateral thyroid lobe & $1(16.67 \%)$ & $3(10.34 \%)$ & $10(34.48 \%)$ \\
Central compartment & $2(33.33 \%)$ & $4(13.79 \%)$ & 0.448 \\
\hline LN-prRLN & $0(0 \%)$ & $6(20.69 \%)$ & 0.515 \\
Others & $2(33.33 \%)$ & $16(55.14 \%)$ & 1.000 \\
\hline Lateral compartment & $3(50.0 \%)$ & & \\
\hline
\end{tabular}

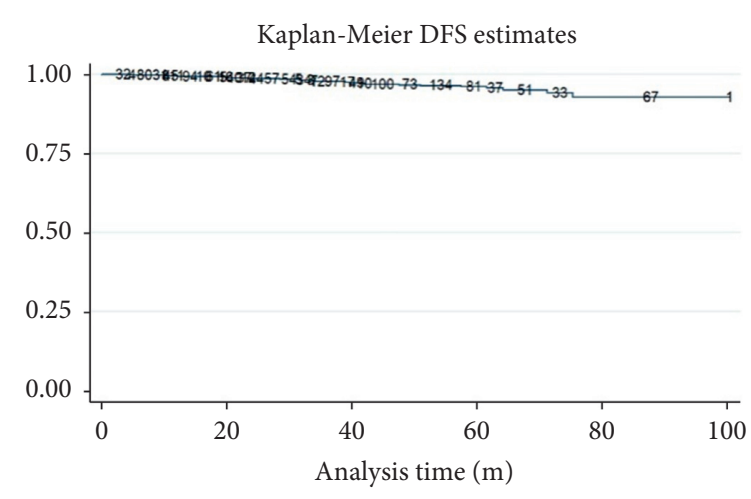

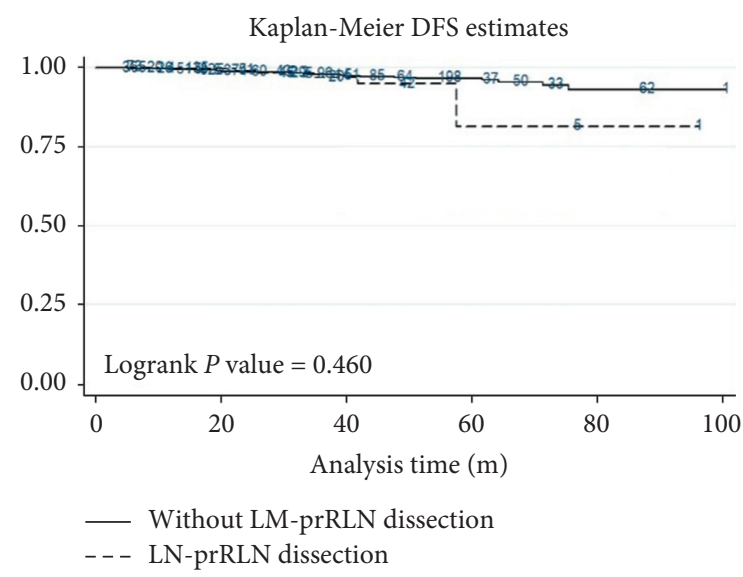

(b)

FIGURE 1: Kaplan-Meier DFS estimates in all patients (a) and the different groups (b).

compartment during follow-up. Complete dissection was performed during the operation. The function of the recurrent laryngeal nerve was good, and there was no residual in the operation area.

\section{Discussion}

PTCs commonly metastasis to the cervical lymph nodes, which primarily occurs in the central compartment lymph nodes. The role of central lymph node dissection in preventing lymph node recurrence has been controversial, especially for patients with negative clinical lymph node metastasis (LNM) (cN0) [15]. However, recent persuasive research suggests that insufficient central lymph node dissection (CLND) after initial surgery is the common cause of recurrence in PTC cases. Therefore, a thorough dissection of the lymphatic tissue in the central compartment may reduce the risks of recurrent or persistent disease by eliminating residual subclinical LNM. Under such circumstances, attention needs to be directed toward the clinical significance of LN-prRLN, which has been frequently unrecognized during right CLND. However, given the different courses along which the RLN runs, to the left or right side, the LN-prRLN has close relationships with the right RLN, esophagus, and prevertebral fascia. Incomplete dissection of these nodes can be a cause of disease recurrence, and reoperation may increase patient morbidity and postoperative complications [16]. Nevertheless, the surgical 
TABLE 7: The demographic characteristics of the four patients with recurrence in LN-prRLN.

\begin{tabular}{|c|c|c|c|c|c|}
\hline Variables & Case 1 & Case 2 & Case3 & Case 4 & Sum \\
\hline Age $(y)$ & 55 & 62 & 29 & 22 & $42.0 \pm 19.48$ \\
\hline Sex (Female/Male) & $\mathrm{F}$ & $\mathrm{F}$ & $\mathrm{F}$ & $\mathrm{F}$ & $4 / 0$ \\
\hline Multifocality (yes/No) & $\mathrm{Y}$ & $\mathrm{Y}$ & $\mathrm{Y}$ & $\mathrm{N}$ & $3 / 1$ \\
\hline Size $(\mathrm{mm})$ & 35 & 12 & 55 & 15 & $29.25 \pm 19.97$ \\
\hline Extensive invasion (yes/No) & $\mathrm{Y}$ & $\mathrm{N}$ & $\mathrm{N}$ & $\mathrm{N}$ & $1 / 3$ \\
\hline Co-HT (yes/No) & $\mathrm{N}$ & $\mathrm{Y}$ & $\mathrm{N}$ & $\mathrm{N}$ & $1 / 3$ \\
\hline Co-nodular goiter (yes/No) & $\mathrm{N}$ & $\mathrm{N}$ & $\mathrm{N}$ & $\mathrm{N}$ & $0 / 3$ \\
\hline cLNMa (yes/No) & $\mathrm{Y}$ & $\mathrm{Y}$ & $\mathrm{Y}$ & $\mathrm{Y}$ & $4 / 0$ \\
\hline Positive/Dissected cLNMa numbers & $11 / 12$ & $11 / 21$ & $9 / 15$ & $6 / 7$ & - \\
\hline LLNM (yes/No) & $\mathrm{N}$ & $\mathrm{N}$ & $\mathrm{Y}$ & Y & $2 / 2$ \\
\hline Positive/Dissected LLNM numbers & - & - & $7 / 21$ & $33 / 75$ & - \\
\hline Follow-up (months) & 31.47 & 33.73 & 39.07 & 23.40 & $31.92 \pm 6.51$ \\
\hline
\end{tabular}

Co-HT: concurrent Hashimoto's thyroiditis; Conodular goiter: concomitant with nodular goiter; cLNMa: central lymph nodes anterior to the right recurrent laryngeal nerve; LLNM: lateral lymph node metastasis.

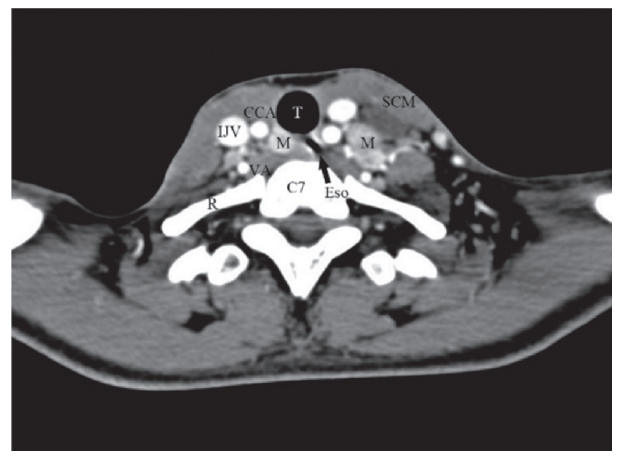

(a)

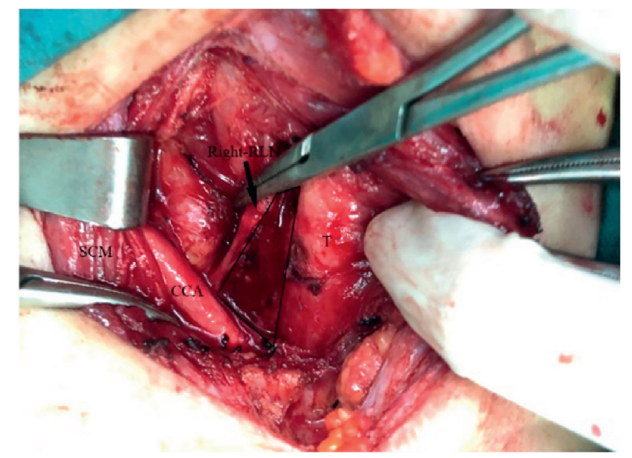

(b)

Figure 2: Axial contrast-enhanced CT of LN-prRLN in the cervical space. Axial contrast-enhanced CTscan shows that the LN-prRLN (M) is medial-posterior to the level of the CCA and IJV and anterior to the prevertebral fascia (aside the tracheoesophageal sulcus). B. The surgical field after LN-prRLN dissection during right-sided central compartment dissection. The part within the quadrangle with solid lines presents the detailed anatomic structure of the LN-prRLNlevel. The right CCA, IJV, and SCM were retracted laterally with a long-arm retractor. CCA: common carotid artery; IJV: interjugular vein; SCM: sternocleidomastoid muscle; T: trachea; VA: vertebral artery; C7: $7^{\text {th }}$ cervical vertebra; R: first rib; Eso: esophagus.

management of lymph node LN-prRLN for PTC remains unclarified. However, the sensitivities of preoperative imaging studies, including ultrasonography and computed tomography, are not sufficient to detect LNM of the central compartment [17]. Thus, risk factors predicting LN-prRLN metastasis should be identified, as they may be valuable to evaluate the nodal status of patients with PTC before surgery and to determine whether lymph node dissection including LN-prRLN is necessary. Consequently, it is imperative to investigate the incidence and predictors of LN-prRLN in PTC.

In the present study, the incidence of cLNM was 55.6\% in group A. However, the incidence of LN-prRLN metastasis was $34.13 \%$ (129 of 378 cases). In some retrospective studies, the LN-prRLN metastasis rates were approximately $11.0 \%$ to 27.2\% [13, 18, 19]. Furthermore, among 1109 patients without LN-prRLN dissection in group B, 29 experienced relapse during the follow-up, of which 4 cases recurred in the location of LN-prRLN, while zero recurred in group A. Considering the rate of LN-prRLN metastasis and recurrence in this compartment, we thought that complete
cLND including LN-prRLN was necessary in the initial surgery treatment.

Tumor size played an important role in stimulating LNprRLN metastasis. Most studies confirmed that a larger tumor led to a greater likelihood of LN-prRLN metastases $[13,20,21]$. In our study, the mean tumor size in patients with and without LN-prRLN metastasis was $13.9 \pm 9.1 \mathrm{~mm}$ vs. $9.6 \pm 6.3 \mathrm{~mm}(P \leq 0.001)$. In multivariate analyses, we also found that there was an association between LNprRLN metastasis and tumor size $(P=0.039)$. These results potently declared that the prevalence of LN-prRLN metastasis rapidly increases with tumor size. It has been reported that $18-87 \%$ of patients with thyroid carcinoma have multiple tumor foci $[22,23]$. In keeping with this, $34.13 \%(129 / 378)$ of patients in our study were multifocal. Several studies have suggested that the presence of multifocality is a risk factor for cLNM in patients with PTC. However, in the present study, we did not observe a significant difference between multifocality and single tumor foci $(1.29(0.83-2.02), P=0.255)$. Since tumors with ETE have a higher degree of malignancy, they are more likely to 
have LNM. In this study, among 36 (9.52\%) patients with ETE, the rate of LN-prRLN metastasis was $16.28 \%$, compared with $6.02 \%$ among those without ETE $(P=0.002)$. However, ETE was not identified as an independent risk factor for LN-prRLN metastasis in the multivariable analysis (1.63 (0.70-3.84), $P=0.258)$. This is consistent with findings reported by Yuan et al. [9] and Bae et al. [8].

Few researchers have examined metastases with concurrent HT or concurrent benign nodular goiters. In this study, we calculated the statistics for HT and nodular goiter according to postoperative pathology. The results showed that LN-prRLN metastases were found in 36 (27.91\%) patients with HT and 104 (39.08\%) patients without HT. The metastatic rate was significantly higher among patients without HT $(P=0.009)$. Although HT is often accompanied by enlarged lymph nodes in the central compartment region, these lymph nodes are often not malignant metastases, including LN-prRLN. Furthermore, HT provided a protective mechanism to reduce the metastatic effect. Otherwise, this phenomenon was not found in patients with nodular goiter.

The presence of right cLNM is significantly associated with LN-prRLN metastasis. Most studies have shown that right cLNM is an independent risk factor for LN-prRLN metastasis $[10,11,13]$. In our study, the proportion of LNprRLN metastasis in the LN-prRLNM- group was substantially lower than that in the LN-prRLNM+ group (41.37\% vs $82.95 \%, P \leq 0.001)$. Multivariate analysis also confirmed that the presence of right CLNM is an independent risk factor for LN-prRLN metastasis. Furthermore, with the increasing number of metastatic right central lymph nodes, the presence of LN-prRLN metastasis also increased substantially. Furthermore, we did not find that the diameter of cLNM was associated with LN-prRLN metastasis. Similarly, a univariate analysis showed that LLNM was also significantly associated with LN-prRLN metastasis.

Our study has a limitation that should be taken into consideration. There was selection bias in these two groups. In the early stage of our center, not all patients underwent LN-prRLN dissection, but patients were young and had a larger tumor size, concurrent Hashimoto's thyroiditis or LNM. These differences were demonstrated in the comparison of group A and group B (Table 2). This retrospective study made us realize that a larger tumor size and positive LNM are real risk factors, and these groups of patients should be treated with LN-prRLN dissection. Therefore, a prospective randomized controlled trial is needed in future studies.

In conclusion, our study revealed that patients with PTC with large tumor sizes, central LN metastasis, and lateral cervical lymph node metastasis are at high risk of LN-prRLN metastasis, and these findings could assist surgeons in evaluating surgical treatment strategies. These findings provide support for the necessity of LN-prRLN dissection in such patients.

\section{Data Availability}

The datasets used to support the findings of this study are restricted by the Ethics Committees of Fudan University Shanghai Cancer Center, in order to protect patient privacy. The data are available from the corresponding author for researchers upon reasonable request.

\section{Disclosure}

Dezhi Wang and Yunjun Wang contributed equally to this study and should be regarded as co-first authors.

\section{Conflicts of Interest}

The authors declare that they have no conflicts of interest.

\section{Authors' Contributions}

Kai Guo and Tuanqi Sun conceptualized and designed the study. Dezhi Wang and Lili Chen collected and assembled the data. Yunjun Wang and Dezhi Wang analyzed and interpreted the data. Yunjun Wang wrote the manuscript. All authors read and approved the final version of the manuscript.

\section{Acknowledgments}

This study was supported by the Shanghai Science and Technology Committee (STCSM) (18411969000).

\section{References}

[1] W. Chen, R. Zheng, H. Zeng et al., "Annual report on status of cancer in China, 2011," Chinese Journal of Cancer Research, vol. 27, pp. 2-12, 2015.

[2] N. Howlader, A. M. Noone, M. Krapcho et al., SEER Cancer Statistics Review, National Cancer Institute, Bethesda, MD, USA, 2016, https://seer.cancer.gov/csr/1975_2013/.

[3] D. S. Cooper, G. M. Doherty, B. R. Haugen et al., "Revised american thyroid association management guidelines for patients with thyroid nodules and differentiated thyroid cancer," Thyroid, vol. 19, pp. 1167-1214, 2009.

[4] O. Gimm, F. W. Rath, and H. Dralle, "Pattern of lymph node metastases in papillary thyroid carcinoma," British Journal of Surgery, vol. 85, no. 2, pp. 252-254, 1998.

[5] A. Machens, R. Hinze, O. Thomusch et al., "Pattern of nodal metastasis for primary and reoperative thyroid cancer," World Journal of Surgery, vol. 26, no. 1, pp. 22-28, 2002.

[6] J. A. Sipos and E. L. Mazzaferri, "Thyroid cancer epidemiology and prognostic variables," Clinical Oncology, vol. 22, no. 6, pp. 395-404, 2010.

[7] S. E. Carty, D. S. Cooper, G. M. Doherty et al., "Consensus statement on the terminology and classification of central neck dissection for thyroid cancer," Thyroid, vol. 19, pp. 1153-1158, 2009.

[8] S. Y. Bae, J. H. Yang, M. Y. Choi, J. H. Choe, J. H. Kim, and J. S. Kim, "Right paraesophageal lymph node dissection in 
papillary thyroid carcinoma," Annals of Surgical Oncology, vol. 19, pp. 996-1000, 2012.

[9] J. R. Yuan, J. H. Li, X. Y. Chen et al., "Predictors of lymph nodes posterior to the right recurrent laryngeal nerve metastasis in patients with papillary thyroid carcinoma," Medicine, vol. 96, no. 35, p. e7908, 2017.

[10] L. Zhang, H. Liu, Y. Xie et al., "Risk factors and indication for dissection of right paraesophageal lymph node metastasis in papillary thyroid carcinoma," European Journal of Surgical Oncology (EJSO), vol. 42, no. 1, pp. 81-86, 2016.

[11] H. Chang, R. N. Yoo, S. M. Kim et al., "The clinical significance of the right paraoesophageal lymph nodes in papillary thyroid cancer," Yonsei Medical Journal, vol. 56, no. 2, pp. 1632-1637, 2015.

[12] Y. S. Kim and W. C. Park, "Clinical predictors of right upper paraesophageal lymph node metastasis from papillary thyroid carcinoma," World Journal of Surgical Oncology, vol. 10, no. 1, p. 164, 2012.

[13] Z. Liu, M. Sun, Y. Xiao et al., "Predictors of metastasis to lymph nodes posterior to the right recurrent laryngeal nerve in differentiated thyroid carcinoma: a prospective study," Asian Journal of Surgery, vol. 40, no. 4, pp. 270-277, 2016.

[14] C. Li, J. Xiang, and Y. Wang, "Risk factors for predicting lymph nodes posterior to right recurrent laryngeal nerve (LNprRLN) metastasis in thyroid papillary carcinoma: a metaanalysis," International Journal of Endocrinology, vol. 2019, Article ID 7064328, 11 pages, 2019.

[15] V. I. Forest, J. R. Clark, A. Ebrahimi et al., "Central compartment dissection in thyroid papillary carcinoma," Annals of Surgery, vol. 253, no. 1, pp. 123-130, 2011.

[16] M. P. Young, M. L. Sang, D. W. Kim, S.-C. Shin, and B.-J. Lee, "Predictive factors of right paraesophageal lymph node metastasis in papillary thyroid carcinoma: single center experience an meta-analysis," PLoS ONE, vol. 12, no. 5, Article ID e0177956, 2017.

[17] E. Kim, J. S. Park, K. R. Son, J. H. Kim, S. J. Jeon, and D. G. Na, "Preoperative diagnosis of cervical metastatic lymph nodes in papillary thyroid carcinoma: comparison of ultrasound, computed tomography, and combined ultrasound with computed tomography," Thyroid, vol. 18, pp. 411-418, 2008.

[18] Z. Pinyi, Z. Bin, B. Jianlong et al., "Risk factors and clinical indication of metastasis to lymph nodes posterior to right recurrent laryngeal nerve in papillary thyroid carcinoma: a single-center study in China," Head Neck, vol. 36, pp. 13351342, 2014.

[19] P. Zhang, B. Zhang, J. Bu et al., "Prospective analysis of the risk factors and clinical indications of dissection of lymph node posterior to right recurrent laryngeal nerve in 283 cases of papillary thyroid carcinoma," Chinese Clinical Oncology, vol. 36, pp. 109-114, 2014.

[20] Z. Pinyi, Z. Bin, B. Jianlong, L. Yao, and Z. Weifeng, "Risk factors and clinical indication of metastasis to lymph nodes posterior to rRLN in papillary thyroid carcinoma: a singlecenter study in China," Head Neck, vol. 36, pp. 1335-1342, 2014.

[21] Y. Ito, M. Fukushima, T. Higashiyama et al., "Incidence and predictors of right paraesophageal lymph node metastasis of N0 papillary thyroid carcinoma located in the right lobe," Endocrine Journal, vol. 60, no. 3, pp. 389-392, 2013.

[22] T. M. Shattuck, W. H. Westra, P. W. Ladenson, and A. Arnold, "Independent clonal origins of distinct tumor foci in multifocal papillary thyroid carcinoma," New England Journal of Medicine, vol. 352, no. 23, pp. 2406-2412, 2005.
[23] S. Y. Park, Y. J. Park, Y. J. Lee et al., "Analysis of differential BRAF(V600E) mutational status in multifocal papillary thyroid carcinoma: evidence of independent clonal origin in distinct tumor foci," Cancer, vol. 107, pp. 1831-1838, 2006. 